\title{
Comparative Study of Immobilized Phthalocyanines in Oxidative Degradation
}

\author{
Péter Bata, András Demjén, Ferenc Notheisz and Ágnes Zsigmond*
}

Department of Organic Chemistry, University of Szeged, Dóm tér 8, 6720-Szeged, Hungary

\begin{abstract}
Immobilized iron phthalocyanine catalysts were prepared, via two heterogenization methods: anchoring by Augustine's method and the preparation of a "ship-in-a-bottle" type catalyst by intrazeolite synthesis method. The heterogenized catalysts were applied in the oxidative destruction of 4-chlorophenol, as a common pollutant of waste water. The performance of the two heterogenized and the homogeneous complexes were compared and it was found that the immobilized complexes had about the same activity as the homogeneous analog. Both heterogenized samples could be recycled in three subsequent runs and the "ship-in-a-bottle" type showed stable activity. Meanwhile, the anchored complex lost some activity in every subsequent run. Consequently, the first catalyst was better for practical application.
\end{abstract}

Keywords: Iron phthalocyanine complex, immobilization, oxidation, degradation of 4-chlorophenol, recyclability.

\section{INTRODUCTION}

Environmental protection is a key issue of today's chemistry research [1]. Inside of this issue the degradation and/or removal of industrial pollutants, such as phenols and its derivatives are essential [2]. Since these compounds are industrially important chemicals, their presence in the environment is relatively common. Due to their high toxicity, they represent a group of dangerous chemicals even at low concentrations. Chlorinated phenols are also produced, whenever chlorination treatment of drinking water is performed. In such cases, trace levels of phenol are transformed into chlorinated derivatives representing a specific risk especially for prolonged exposures. The destruction of these derivatives is essential from an environmental point of view [2].

Macrocyclic compounds known as phthalocyanines (Pc's) were first described in the 1930's [3]. Since then, many interesting applications have appeared due to their unique physical and chemical properties [4]. Due to their stability and light absorption in characteristic regions of the visible part of the spectrum, phthalocyanines have been used as pigments and dyes. Recently other applications based on their distinctive property to generate an active species upon light absorption, have appeared, as well. These photoinduced events may occur due to the ability of some phthalocyanines to excite ground-state molecular oxygen $\left({ }^{3} \mathrm{O}_{2}\right)$ to highly active singlet oxygen $\left({ }^{1} \mathrm{O}_{2}\right)$ [5]. Singlet oxygen is a very efficient oxidant, which degrades 4chlorophenol effectively [6,7]. However, only phthalocyanines containing transition metal ions with a closed valence shell, such as $\mathrm{Al}^{3+}, \mathrm{Zn}^{2+}$ are efficient as photocatalysts [8]. The phthalocyanines, which are inactive

*Address correspondence to this author at the Department of Organic Chemistry, University of Szeged, Dóm tér 8, 6720-Szeged, Hungary;

Tel: +36-62-544 512; Fax: +36-62-544 200;

E-mail: azsig@chem.u-szeged.hu in photocatalysis, can also be used as oxidation catalysts in dark, together with common oxidation agents: hydrogen peroxide, peroxyacetic acid and tert-butylhydroperoxide $(t$ $\mathrm{BuOOH})$. This approach is considered as an alternative to the decomposition of various environment endangering chemicals with the singlet oxygen based mechanism [9]. Referring to the environmental application of Pcs's, it is important to note that they are virtually non-toxic and they have relatively simple preparation method even on a large scale.

The strategy - the heterogenization of metal complexes has about 30 years of history but the increasing importance of selectivity resulted in a new renaissance of this field [10]. Several different strategies were introduced to immobilize metal complexes, which can be divided into two basic directions: 1. to built the complex inside a porous support $[11,12] 2$. to graft into the surface of a support $[13,14]$. The performance of the immobilized complexes were compared to the performance of the homogeneous analogues and usually the heterogenized complexes had higher activity and selectivity. Further, the easy handling and the recyclability make them favourable as compared to their homogeneous counterpart $[15,16]$.

The major intention of this contribution was to present results on the application of immobilized iron phthalocyanine, as an oxidation catalyst in dark, together with $t$-BuOOH. 4-chlorophenol was used as model pollutant, to represent a dangerous contamination. The heterogenized iron phthalocyanine catalysts were prepared in two different ways, characterized by spectroscopic techniques and applied in the oxidative degradation of 4-chlorophenol.

\section{MATERIALS AND METHODS}

\subsection{Catalysts}

Iron phthalocyanine was prepared using solvent free condition, applying microwave irradiation. Here, only brief information is given, details are described elsewhere $[17,18]$. 
$2.5 \mathrm{~g}$ of iron(II) chloride $(0.02 \mathrm{~mol})$ and $10.25 \mathrm{~g}$ phthalonitrile $(0.08 \mathrm{~mol})$ was used for the preparation. The homogeneous mixture of the two components was heated in a beaker for 6 minutes in a microwave oven (an intensive blue color was developed). After cooling the reaction mixture to room temperature, the complex was crushed to a fine powder and dissolved in $20 \mathrm{ml}$ of acetone. The mixture was refluxed for 30 minutes and the remaining powder was filtered and dried. With this treatment the uncomplexed ligand was removed. In order to get a higher and constant activity, another washing with cc. $\mathrm{H}_{2} \mathrm{SO}_{4}$ was applied and the final catalyst was a deep blue fine power.

The anchoring of the complex was done in two steps, by a method developed by Augustine et al. $[13,14,16] .1 .5 \mathrm{~g}$ of $\mathrm{Al}_{2} \mathrm{O}_{3}$ was suspended in $30 \mathrm{~mL}$ of methanol. $386.7 \mathrm{mg}(0.15$ mmol) of phosphotungstic acid hydrate was dissolved in 25 $\mathrm{mL}$ of methanol and this solution was added dropwise into the alumina suspension under efficient stirring. The stirring was continued for one day at room temperature, under an $\mathrm{Ar}$ atmosphere. The solid residue was filtered and suspended in $30 \mathrm{~mL}$ of methanol. $56.8 \mathrm{mg}(0.1 \mathrm{mmol})$ of iron phthalocyanine complex was dissolved in $40 \mathrm{~mL}$ of deoxygenated methanol and this solution was dropped slowly with stirring to the suspension. The stirring was continued for another day. The solution was removed and the solid residue was washed with methanol, until a colorless solution was obtained. The light blue solid was dried at $30{ }^{\circ} \mathrm{C}$ for two hours in vacuum and for one day under argon. $1.5 \mathrm{~g}$ of catalyst was obtained.

The zeolite-encapsulated iron phthalocyanine was prepared by the intrazeolite synthesis method under Ar [12]. $\mathrm{Fe}^{2+}$-containing zeolite were prepared by ion exchange for 3 $\mathrm{h}$ at $50{ }^{\circ} \mathrm{C}$, using $2 \mathrm{~g}$ of $\mathrm{NaY}$ zeolite and $40 \mathrm{ml}$ of $1 \mathrm{~N} \mathrm{FeSO}_{4}$ $x 7 \mathrm{H}_{2} \mathrm{O}$. The ion exchanged zeolite was filtered, washed with deionized water, until no iron was observed in the filtrate, and with methanol and finally dried under Ar. The $\mathrm{Fe}^{2+}$-containg zeolite was mixed with the mixture of $2 \mathrm{~g}$ phthalonitrile and $3 \mathrm{~g}$ diphenil and was heated at $423 \mathrm{~K}$ for 4 hours under Ar, then cooled to room temperature. The product was extracted with acetone in a Soxhlet extractor for $48 \mathrm{~h}$ to remove the excess of the precursor and all the possible complex, which was formed on the zeolite surface. The solid was stirred with saturated $\mathrm{NaCl}$ solution for $24 \mathrm{~h}$ at room temperature to exchange the uncomplexed $\mathrm{Fe}^{2+}$ ions. Finally it was dried overnight and 2,07 $\mathrm{g}$ light blue catalyst was obtained.

\subsection{Catalysts Characterization}

The presence of immobilized and encapsulated complexes was demonstrated by FT-IR spectroscopy [19]. The FT - IR spectra of the zeolite, the neat complexes and the heterogenized samples were taken, as well. The spectra were recorded in $\mathrm{KBr}$ pellets, using Bio-Rad FTS - $65 \mathrm{~A}$ spectrophotometer, in the range of $400-4000 \mathrm{~cm}^{-1}$.

XRD spectra of the supports were also taken [20] and the metal content was determined by ICP-AES. The XRD spectra were recorded on a Philips PW - 1830 diffractometer. To determine the metal content, the sample was dissolved in cc. $\mathrm{HNO}_{3}$. The metal contents of the solutions were determined by JOBLIN YVON 24 type ICP-AES instrument.

\subsection{Oxidation Reaction}

Catalytic experiments were carried out in a glass vessel containing a magnetic stirrer, at room temperature and atmospheric pressure. In a typical experiment $50 \mathrm{ml}$ of 4chlorophenol/water solution with $2 \times 10^{-4} \mathrm{~mol} / 1$ concentration $\left(\mathrm{n}_{\mathrm{Ph}}=1 \times 10^{-5} \mathrm{~mol}\right)$ and the amount of iron phthalocyanine 5 $\mathrm{mg}\left(\mathrm{n}_{\mathrm{Pc}}=8.8 \times 10^{-6} \mathrm{~mol}\right)$ or $150 \mathrm{mg}$ of immobilized iron phthalocyanine complex were used. $0.2 \mathrm{ml}\left(1.4 \times 10^{-3} \mathrm{~mol}\right) t$ $\mathrm{BuOOH}$ was applied in each experiment, and the reaction proceeded with continuous stirring. The reaction vessel was carefully covered with black foil to prevent any contact of the complex with light.

Samples were taken at a given time and analyzed by HPLC using RP-18 type of column. Methanol to water mobile phase ratio 50:50 was set up and for the detection wavelength $282 \mathrm{~nm}$ was chosen.

The oxidation of 4-chlorophenol occurs via a well know mechanism [6] involving several intermediates, of which $p$ benzoqinone is predominant. Depending on the conditions $(\mathrm{pH})$, futher oxidation can happen, resulting maleic and fumaric acids.

\section{RESULTS AND DISCUSSION}

With the aim of developing active and recyclable catalysts, we have prepared immobilized iron phthalocyanine catalysts via different heterogenization ways: the complex was anchored onto the surface of alumina and built inside $\mathrm{NaY}$ zeolite by intrazeolite complexation method. The heterogenized catalysts were characterized by spectroscopic methods and applied in the oxidative degradation of 4chlorophenol. The performance of the immobilized catalysts and the homogeneous complex were compared and the best recyclable catalyst was selected.

\subsection{Catalysts Characterization}

The FT-IR spectra of the supports $\left(\mathrm{Al}_{2} \mathrm{O}_{3}\right.$ and $\mathrm{NaY}$ zeolite), the iron phthalocyanine and the heterogenized samples were taken. Fig. (1) shows the FT-IR spectra of $\mathrm{Fe}(\mathrm{Pc})$ and $\mathrm{Fe}(\mathrm{Pc}) /$ zeolite and the spectra of $\mathrm{NaY}$ zeolite.

The comparison of these spectra proved that the iron phthalocyanine complex was anchored to the surface of alumina and built inside the zeolite. The characteristic bands for the iron phthalocyanine like $(1515,1494,1333,1164$, 1118 and $752 \mathrm{~cm}^{-1}$ ) appeared in the spectra of the immobilized samples, as well. As the matter of fact, a slight shift, a couple of $\mathrm{cm}^{-1}$ was observed in the spectra of the immobilized complexes to prove that the iron phthalocyanine complex was really anchored into the surface or built inside the zeolite. The slight shift caused by the small changes in the structure of the complex during the grafting to the surface or building inside the zeolite [21].

The XRD diffractograms of the zeolite and the "ship-ina-bottle" complex were taken as well (Fig. 2).

The comparison of these spectra showed no change in the zeolite structure as a consequence of the complex formation.

In order to determine the complex concentration of the immobilized samples, the samples were dissolved in cc. $\mathrm{HNO}_{3}$ and the metal content of these solution were determined by ICP-AES method. As we proved with an 


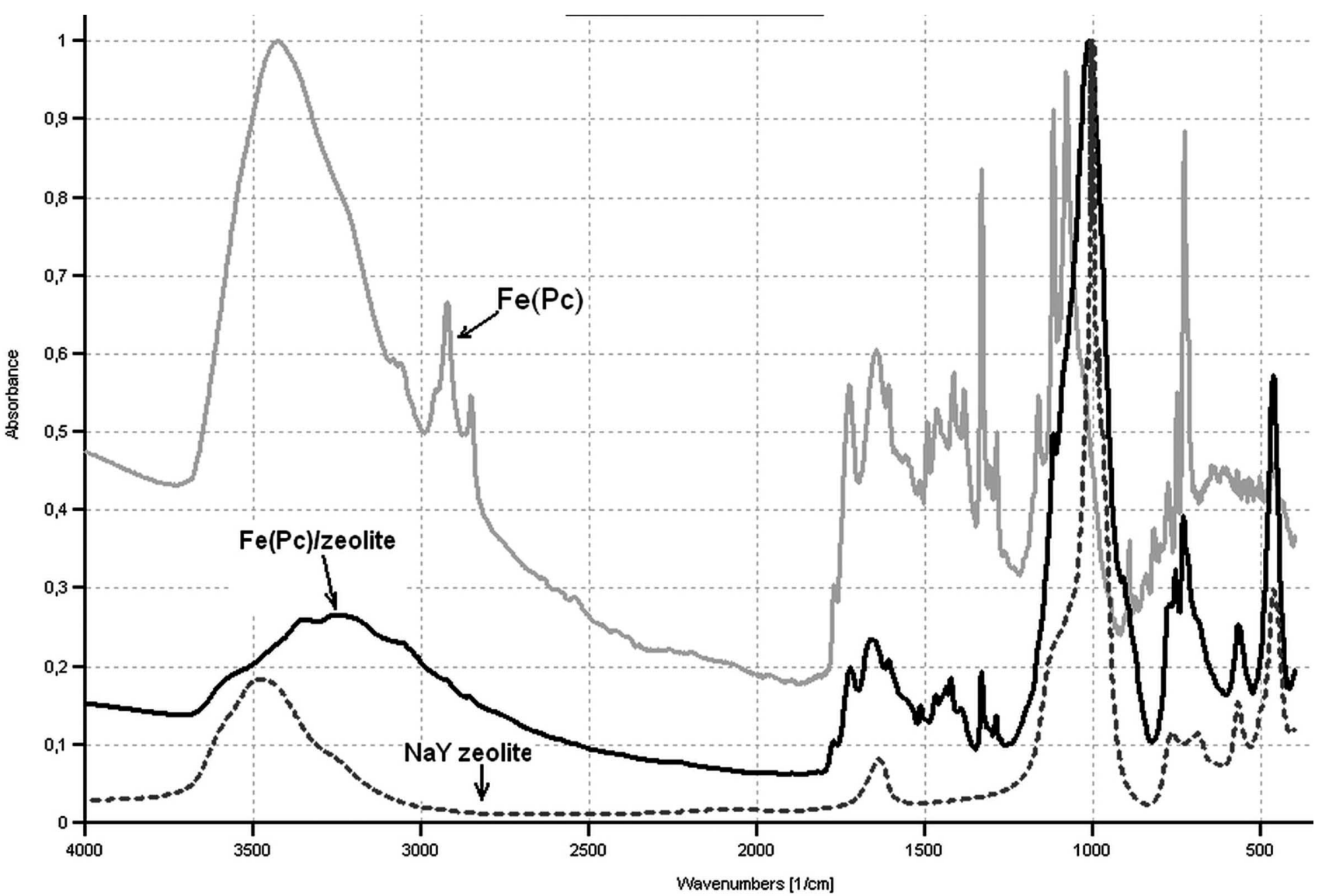

Fig. (1). The FT-IR spectra of $\mathrm{Fe}(\mathrm{Pc})$ and $\mathrm{Fe}(\mathrm{Pc}) / \mathrm{Z}-\mathrm{NaY}$ zeolite.

additional experiment, the extraction procedure removed all the amount of complex from the zeolite surface. Thus, all the residual iron can be found in the zeolite cages and may be associated with the amount of encapsulated $\mathrm{Fe}(\mathrm{Pc})$. The anchored catalyst was found to contain $35.8 \mu \mathrm{mol} \mathrm{Fe} / \mathrm{g}$ catalyst and $42.0 \mu \mathrm{mol} \mathrm{Fe} / \mathrm{g}$ in the case of the "ship-in-abottle" catalyst.

\subsection{Oxidation Reaction}

The oxidative degradation of 4-chlorophenol was studied in dark, using iron phthalocyanine catalysts and $t$-BuOOH, as an oxidant. During this study we have compared the performance of the homogenous and the immobilized catalysts. The initial 4-chlorophenol concentration was $2 \times 10^{-4}$ $\mathrm{mol} / \mathrm{l}$, the decrease of this concentration was followed.

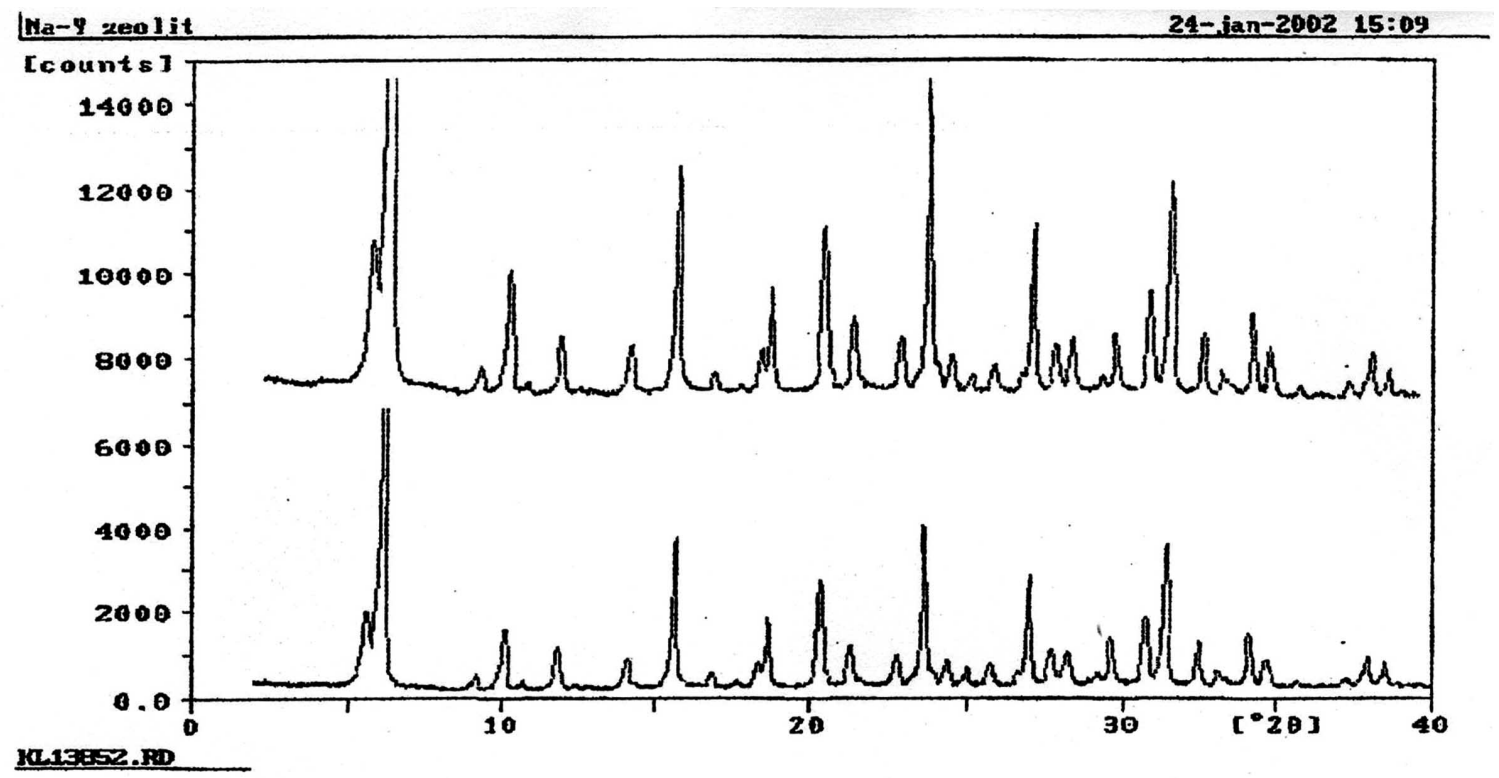

Fig. (2). The XRD diffractogram of NaY zeolite before and after the encapsulation. 


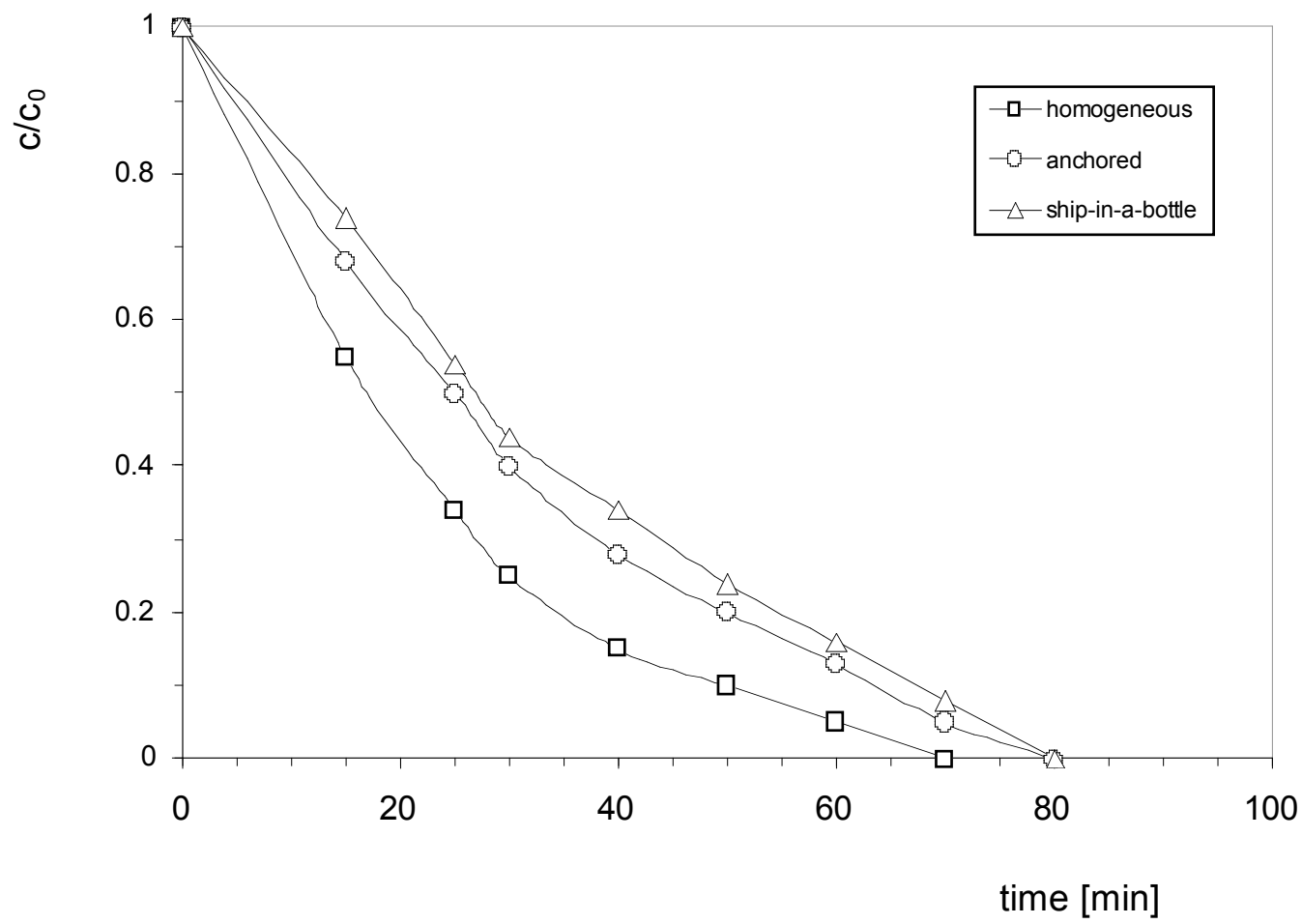

Reaction conditions: T: $25^{\circ} \mathrm{C}, 50 \mathrm{ml}$ 4- chlorophenol/water solution $2 \times 10^{-4} \mathrm{~mol} / \mathrm{l}$ conc., $\mathrm{Fe}(\mathrm{Pc}) 5 \mathrm{mg}, \mathrm{Fe}(\mathrm{Pc}) / \mathrm{hordozó}: 150 \mathrm{mg}, 0.2 \mathrm{ml}$ t-BuOOH

Fig. (3). The decrease of 4-chlorophenol concentration using homogeneous and two immobilized iron phthalocyanines catalysts.

Samples were taken every 15 minutes and the concentration was determined by HPLC (Fig. 3).

Fig. (3) clearly shows that both immobilized complexes were active in the oxidative degradation of 4-chlorophenol. Although the decrease in the concentration is a little bit slower on the heterogenized catalysts than in the homogeneous one, the time necessary for achieving nearly $100 \%$ conversion is comparable. We can also compare the reaction rates on the active sites in each case. Having determined the amount of complex on the immobilized samples, we can calculate the specific rate at $15 \mathrm{~min}$ and compare the TOF's (TOF $=$ transferred 4-chlorophenol (mol)/used catalyst (mol) x 1 (hour)) (Table 1).

Table 1. The TOF of the Oxidative Degradation of 4Chlorophenol in Homogenous and Heterogenized Condition

\begin{tabular}{|c|c|c|c|}
\hline Catalysts & $\mathbf{F e}(\mathbf{P c})[\mathbf{m o l}]$ & Degradation [\%] & TOF $\left[\mathbf{h}^{-1}\right]$ \\
\hline \hline $\mathrm{Fe}(\mathrm{Pc})$ & $8.8 \times 10^{-6}$ & 45 & 2.04 \\
\hline $\mathrm{FePc} / \mathrm{Z}$ & $6.3 \times 10^{-6}$ & 26 & 1.65 \\
\hline $\mathrm{FePc} / \mathrm{Al}_{2} \mathrm{O}_{3}$ & $5.4 \times 10^{-6}$ & 32 & 2.38 \\
\hline
\end{tabular}

Reaction conditions: T: $25^{\circ} \mathrm{C}, 50 \mathrm{ml}$ 4- chlorophenol/water solution $2 \times 10^{-4} \mathrm{~mol} / 1 \mathrm{lconc}$., $\mathrm{Fe}(\mathrm{Pc}) 5 \mathrm{mg}, \mathrm{Fe}(\mathrm{Pc}) /$ hordozó: $150 \mathrm{mg}, 0.2 \mathrm{ml} t$-BuOOH .

Comparing the calculated TOF's (at 15 minutes) the following conclusions can be drawn: the difference we have seen in the concentration curves appear in the TOF's as well. The anchored catalyst has a little higher activity than the homogeneous complex, meanwhile the TOF of the encapsulated one somewhat lower. The first observation is in good correlation with our former results [15]. For other systems, we have always observed a higher activity for the immobilized catalysts than for the homogeneous analogue. This is one of the advantages of the immobilized catalyst. However, the results of encapsulated complex is in contradiction with the above observation. Comparing the performance of the two immobilized samples, the trend is similar to that in a different system [12]. Studying the hydrogenation of 2-methylpenthenoic acid on heterogenized $\mathrm{Rh}$ (cyclooctadiene)(L-prolinamide) catalysts (prepared by encapsulation and anchoring on $\mathrm{NaY}$ zeolite) we have observed that the activity of the anchored complex is several times higher than the encapsulated one. In the case of the encapsulated catalyst the complex can be found inside the zeolite supercage, while in case of anchored catalyst the complex is situated on the outer surface of the zeolite. In other words, in case of anchored catalyst the real acive sites are more accessible to the reactant molecules than in the case of the encapsulated catalyst. The observed trend is the same in this case, but the difference is not so explicit as it was in the former study.

\subsection{Catalysts Recycling}

Studying the performance of immobilized catalysts, not only the activity is important but the stability, as well, since the most important advantages of immobilization are the easy handling and recyclability of the immobilized complexes. Consequently we have studied the reusability of our heterogenized samples. The catalysts were simply filtered from the reaction mixture and applied in an additional run without futher purification or activation. Both the immobilized samples were applied in three subsequent runs and the obtained results can be seen on Figs. $(\mathbf{4}, \mathbf{5})$. To 


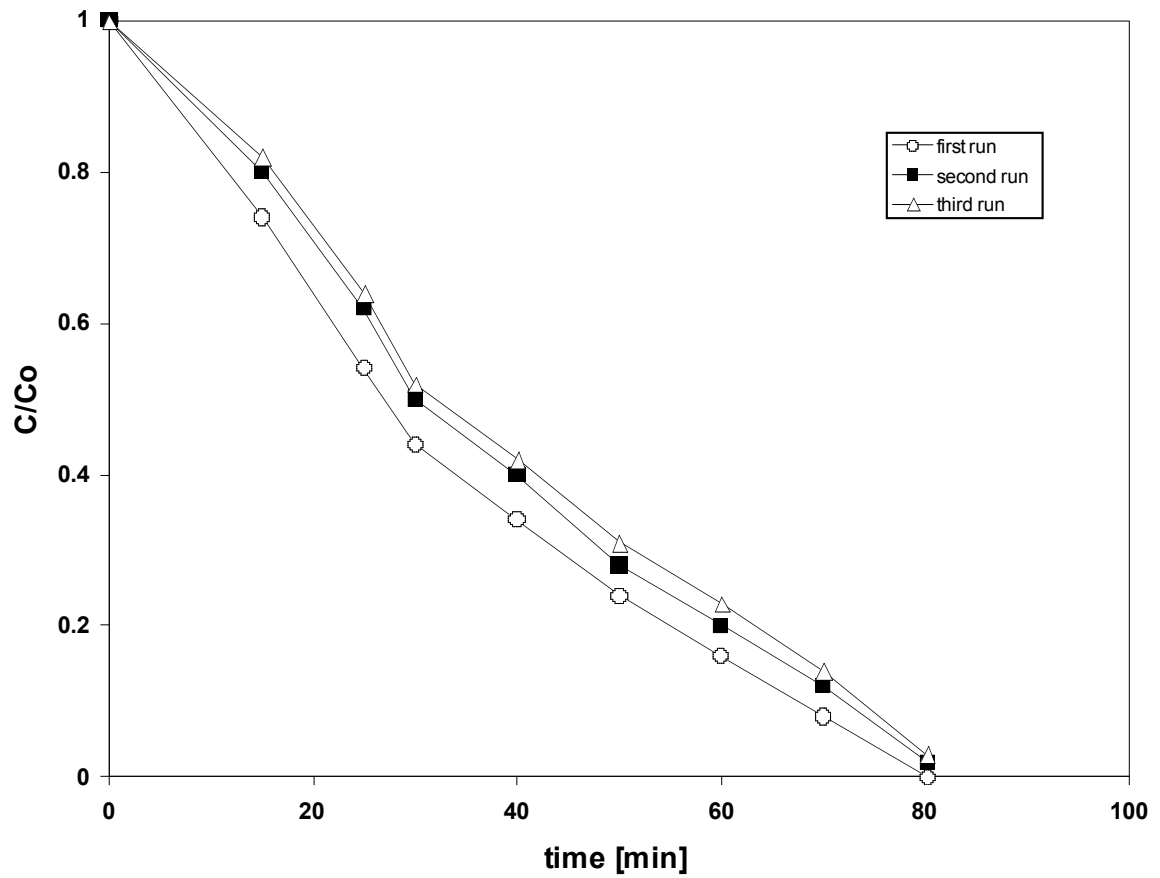

Reaction conditions: T: $25^{\circ} \mathrm{C}, 50 \mathrm{ml}$ 4- chlorophenol/water solution $2 \times 10^{-4} \mathrm{~mol} / \mathrm{l}$ conc., $\mathrm{Fe}(\mathrm{Pc}) / \mathrm{Z}: 150 \mathrm{mg}, 0.2 \mathrm{ml} t$-BuOOH

Fig. (4). The recyclability of the $\mathrm{Fe}(\mathrm{Pc}) / \mathrm{Z}(\mathrm{Fe}(\mathrm{Pc})$ complex in zeolite) catalyst in the oxidative degradation of 4-clorophenol.

have the fair comparison the same protocol was applied in both cases.

As it can be seen on Figs. $(4,5)$, both of the immobilized catalysts were active in three subsequent runs. However the two heterogenized catalyst had different behaviour; while the "ship-in-a-bottle“ type catalysts showed the expectable behaviour, the catalyst, made by the grafting method, was different. The activity of the $\mathrm{Fe}(\mathrm{Pc}) / \mathrm{Z}$ catalyst decreased after the first run, but it was constant in an additional run. This means that the zeolite encapsulated catalyst preserved its activity, while the other immobilized sample - the one, which had the higher TOF- lost activity in every subsequent run.

Considering the stability of the immobilized catalysts, the encapsulated catalyst had a better performance having more or less the same activity. In spite of the fact that the encapsulated complex had a lower TOF but its activity was constant in

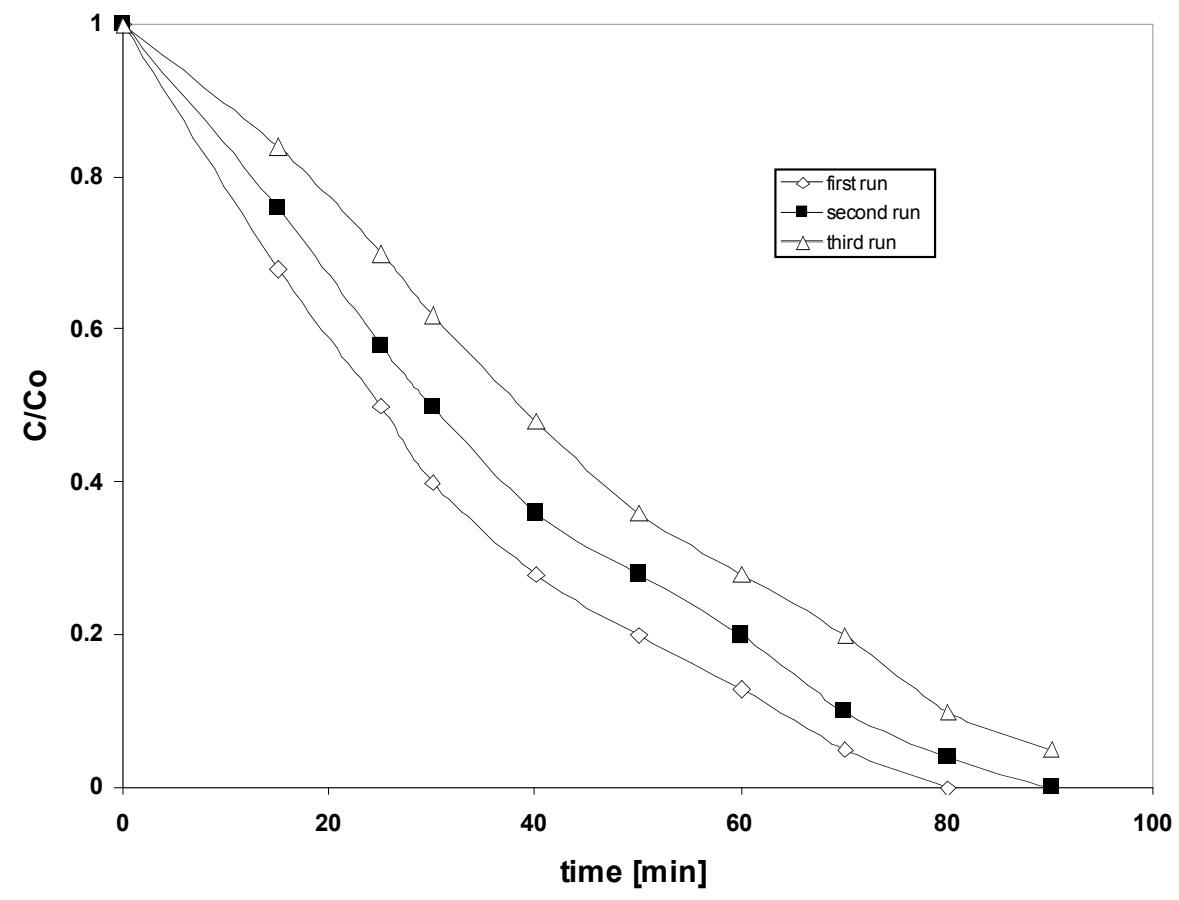

Reaction conditions: $\mathrm{T}: 25^{\circ} \mathrm{C}, 50 \mathrm{ml}$ 4- chlorophenol/water solution $2 \times 10^{-4} \mathrm{~mol} / / \mathrm{conc} ., \mathrm{Fe}(\mathrm{Pc}) / \mathrm{Al}_{2} \mathrm{O}_{3}: 150 \mathrm{mg}, 0.2 \mathrm{ml} t-\mathrm{BuOOH}$

Fig. (5). The reusability of the $\mathrm{Fe}(\mathrm{Pc}) / \mathrm{Al}_{2} \mathrm{O}_{3}$ catalyst in the oxidative degradation of 4-chlorophenol. 
subsequent runs. Meanwhile the activity of the anchored complex descreases in every subsequent run. From the practical point of view, the stability is even more important than the higher TOF, which means that the encapsulated catalyst seems to be a better catalyst.

Studying a similar comparison in the hydrogenation of 2methylpenthenoic acid we have observed a better performance of the anchored catalyst, showing several times higher activity, than the encapsulated one [12]. In this study the higher activity was preserved in three subsequent runs without any significant changes.

In the light of the more or less contradictory results we have to emphasize, that using heterogenized catalysts we have to be very careful to predict the catalyst performance. It depends on the way of preparation, the free complex and the studied reaction as well.

Applying the immobilized samples, leaching was checked in both cases, with the so called Sheldon test: we have filtered out the catalysts and the reaction did not run without catalyst showing there was no significant leaching.

\section{CONCLUSIONS}

The iron phthalocyanine complex was immobilized in two different ways, using encapsulation and anchoring methods. The catalysts were characterized by spectroscopic methods and applied in the oxidative degradation of 4-chlorophenol. The performance of the heterogenized samples were compared to the homogeneous complex and to each other. Both the immobilized samples proved to be active in the above reaction, even in three subsequent runs. Our results showed that the anchored catalyst lost activity in each subsequent run, meanwhile the activity of the encapsulated one - after a small decrease - stabilizes. So, if we want to select the best catalyst for this reaction, we have to consider not only the activity, but the reusability, as well.

\section{ACKNOWLEGDEMENT}

The Project named „TÁMOP-4.2.1/B-09/1/KONV-20100005 - Creating the Center of Excellence at the University of Szeged" is supported by the European Union and co-financed by the European Regional Fund.

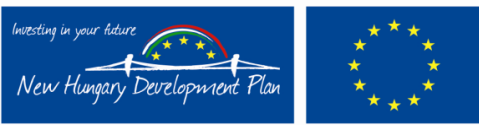

\section{CONFLICT OF INTEREST}

The author(s) confirm that this article content has no conflicts of interest.

\section{REFERENCES}

[1] Lancaster, M. Green Chemistry. Springer - Verlag: Cambrdge, 2002.

[2] Kluson, P.; Drobek, M.; Zsigmond, Á.; Baranyi, J.; Bata, P.; Zarubova, S.; Kalaji, A. Enviromentally friendly phthalocyanine catalysts for water decontamination-Non-photocatalytic systems. Appl. Catal. B., 2009, 91, 605-609.

[3] Linstead, R.P. Phthalocyanines part I. New type of synthetic colouring matter. J. Chem. Soc., 1934, 1016.

[4] Leznoff, C.C.; Lever, A.B.P. Phthalocyanines-Properties and Applications. VCH: Weinheim, New York, 1993.

[5] DeRosa, M.C.; Crutchley, R.J. Photosensitized singlet oxygen and its application. Coord. Chem. Rev., 2002, 351, 233-234.

[6] Kluson, P.; Drobek, M.; Kalaji, A.; Zarubova, S.; Krysa, J.; Rakusan, J. Singlet oxygen photogeneration efficiences of a series of phthalocyanines in a well-defined spectral regions. Photochem. Photobiol. A. Chem., 2008, 199, 267-273.

[7] Kluson, P.; Drobek, M.; Strasak, T.; Krysa, J.; Karaskova, M.; Rakusan, J. Sulphonated phthalocyanines as effective oxidation photocatalysts for visible and UV light regions. J. Mol. Catal. A. Chem., 2007, 272, 213219.

[8] Ozoemena, K.; Kuznetsova, N.; Nyokong, T. Photosensitized transformation of 4-chlorophenol in the presence of aggregated and non-aggregated metallophthalocyanines. J. Photochem. Photobiol. A. Chem., 2001, 139, 217-224.

[9] Kluson, P.; Drobek, M.; Krejcikova, S.; Krysa, J.; Kalaji, A.; Cajthaml, T.; Rakusan, J. Molecular structure effects in photodegradation of phenol and its chlorinated derivatives with phthalocyanines. Appl. Catal. B., 2008, 80, 321-326.

[10] Zsigmond, Á.; Notheisz, F.; Kluson, P.; Floris, T. Heterogenized homogeneous catalysts for fine chemicals production materials and processes. In: Catalysis by Metal Complexes; Barbaro, P.; Liguori, F.; Eds.; Springer: Dordrecht, 2010, Vol. 33, pp. 283-361.

[11] Wöltinger, J.; Bäckvall, J.E.; Zsigmond, Á. Zeolite-encapsulated cobalt salophens as efficient oxygen activating catalysts in palladiumcatalyzed aerobic 1,4-oxidation of 1,3-dienes. Chem. Eur. J., 1999, 5, 1460-1467.

[12] Zsigmond, Á.; Bogár, K. ; Notheisz, F. Comperative study of "ship-ina-bottle" and anchored Rh complexes. J. Catal., 2003, 213, 103-108.

[13] Augustine, R.L.; Tanielyan, S.K.; Anderson, S.; Yang, H. A new technique for anchoring homogeneous catalysts. Chem. Commun., 1999, $1257-1258$

[14] Augustine, R.L.; Tanielyan, S.K.; Mahata, N.; Gao, Y.; Zsigmond, Á.; Yang, H. Anchored homogeneous catalysts: the role of the heteropoly acid anchoring agent. Appl. Catal. A. Gen., 2003, 256, 69-76

[15] Zsigmond, Á.; Notheisz, F. Selective synthesis on heterogenized metal complexes. Curr. Org. Chem., 2006, 10, 1655-1680.

[16] Zsigmond, Á.; Balatoni, I.; Notheisz, F.; Herseczky, Zs.; Bakos, J. New, efficient, heterogenized catalysts for asymmetric hydrogenations of prochiral olefins. Catal. Lett., 2005, 101, 195-199.

[17] Lowery, M.K.; Starshak, A.J.; Esposito, J.N.; Krueger, P.C.; Kenney, M.E. Dichloro(phthalocyanino)silicon. Inorg. Chem., 1965, 4, 128-132.

[18] Kluson, P.; Drobek, M.; Kalaji, A.; Karaskova, M.; Rakusan, J. Preparation, chemical modification and absorption properties of various phthalocyanines. Res. Chem. Intermediates, 2009, 35, 103-116.

[19] Sabater, M.J.; Corma, A.; Domenech, A.; Fornes, V.; Gracia, H. Chiral salen magnase complex encapsulated within zeolite $\mathrm{Y}$ : a heterogeneous enantioselective catalyst for the epoxidation of alkenes. J. Chem. Soc. Chem. Commun., 1997, 1285-1286.

[20] Zsigmond, Á.; Notheisz, F.; Bäckvall, J.E. Rate enhancement of oxidation reactions by the encapsulation of metal phthalocyanine complexes. Catal. Lett., 2000, 65, 135-139.

[21] De Vos, D.E. ; Jacobs, P.A. Heterogenization of Mn an Fe complex oxidation catalysts. Catal. Today, 2000, 57, 105-114. 